\title{
THE FUTURE OF ONLINE LEARNING USING ARTIFICIAL INTELLIGENCE
}

\author{
Yew Kee Wong
}

School of Information Engineering, HuangHuai University, Henan, China

\begin{abstract}
Online learning is the emerging technique in education and learning during the COVID-19 pandemic period. Traditional learning is a complex process as learning patterns, approach, skills and performance varies from person to person. Adaptive online learning focuses on understanding the learner's performance, skills and adapts to it. The use of advanced technology also provides a means to analyse the behavioural learning pattern. As it provides the detailed skill mapping and performance which enables the learner to understand the areas needs to be improved. The information can also be used by assessors to improve the teaching approach. Advanced online learning system using artificial intelligence is an emerging concept in the coming years. In this new concept, the classes are not taken face-to-face in a classroom but through an electronic medium as a substitute. These virtual learning approach are gaining importance every day and very soon they are going to be an integral part of our world. Taking up these virtual learning through an electronic medium is termed as online learning. We proposed two new models which are powered by artificial intelligence (AI) tools. A number of examples of using these new models are presented.
\end{abstract}

\section{KEYWORDS}

Analysis Algorithm, Artificial Intelligence, Hybrid Integrated Model, Online Learning, Progressive Response Learning.

\section{INTRODUCTION}

The purpose of this adaptive and advanced online learning system is to enable learners to get proper knowledge of course and adjusting system according to the users IQ level [1]. It is an educational method which uses computers as interactive teaching devices and reduces the teacher's workload and enhance the learner understanding. As the online learning system integrated with Artificial Intelligence (AI) is a new approach it has many flaws. Learning is a sophisticated process. And if the computers have to replace a human teacher they need to be more intelligent as Creative Intelligence Learning (CIL) approach does not provide the quantitative teaching learning mechanism [2]. To strengthen the online learning process with the AI mechanism the paper proposed two new adaptive online learning models, 1: Statistical Analysis using AI Learning Model, and 2: Progressive Response Learning Model. This paper will elaborate the models in detail.

\section{Statistical ANALySis USING ARTIFicial INTELLigenCe LEARNing MODEL}

Many online learning assessment systems that use multiple choice approach is based on the correct answers to judge a learner on their understanding of what they have learned [3]. We have

David C. Wyld et al. (Eds): NLPTA, EDU, DSA, IoTE, VLSI, DPPR, ACITY, AIAA, CNDC - 2021

pp. 187-194, 2021. CS \& IT - CSCP 2021

DOI: $10.5121 /$ csit.2021.111915 
carry out various experiments on these quantifiable measurements (assessment indicators) on Mathematics and English subject on different learner groups. With these assessment indicators, assessors and learners can easily assess the online learning performances [4].

In our research, we have identify 3 critical assessment indicators which can influence the learners' learning progress and understanding. These 3 assessment indicators have interrelationships with the underlying final mark from the assessment [5]. At the end of the assessment, the artificial intelligence engine will analyse all the statistical information from these 3 indicators and provide a recommendation for the assessor and learner.

(1). Difficulty Level (measure by the complexity of the questions [6]). Each question will have the difficulty level embedded. For example, we take a topic in Addition from Mathematics subject. For an Addition topic, we can assign the Difficulty level to these 3 questions depending on the complexity, i.e. $4+3=$ ? (Low Difficulty), $755+958=$ ? (Medium Difficulty) and 7,431,398,214 + 32,883,295 = ? (High Difficulty).

\begin{tabular}{|c|c|}
\hline Level Terms & Quantitative Measurement \\
\hline High (hardest) & 3 \\
\hline Medium & 2 \\
\hline Low (easiest) & 1 \\
\hline
\end{tabular}

(2). Understanding Level (measure by the time from the question appear to submission). Each question will have the understanding level embedded. For example, assuming there is a question with Level Term - High (from a to $b$ ) where " $a=3$ seconds" and " $b=5$ seconds". In this example, if the learner can submit the answer between 3 to 5 seconds after the question appeared than the answer will be assigned 2 points for the Understanding indicator.

\begin{tabular}{|c|c|}
\hline Level Terms & Quantitative Measurement \\
\hline High (from a to $b$ ) fastest & 2 \\
\hline Medium (from $b$ to $c$ ) & 1 \\
\hline Low (from c onwards) slowest & 0 \\
\hline
\end{tabular}

(3). Confident Level (variation in choosing an answer before submission).

For each question, we will capture the behaviour of the learner when choosing an answer before submission [7]. For example, for most learners if they are confident and prudent on choosing the correct answer, they will submit the answer once decided without making any changes. If the learner didn't make any changes when answer this example question, than this answer will be assigned 2 points for the Confident indicator.

\begin{tabular}{|c|c|}
\hline Level Terms & Quantitative Measurement \\
\hline High (no change on first pick) & 2 \\
\hline Medium (one change) & 1 \\
\hline Low (two changes or more) & 0 \\
\hline
\end{tabular}


In this research, we have carry out multiple experiments to evaluate the use of assessment indicators and the statistical information generated when the learner performing the assessment [8][9]. We have conducted 3 detail experiments and the outcomes generated shows promising result on the learners' overall learning performances. Below are the 3 experiments summary which we have conducted on 100 online voluntary learners. All marks and indicators have been converted to percentage $(\%)$ prior for further analysis by the AI engine.

Both the assessment indicators and statistical information stand alone do not have any representations and it is meaningless without others being analysed altogether [10][11]. Furthermore, in order to have an effective and efficient online learning outcome for the learner, the assessor requires to design and develop the curriculum, learning materials and Q\&A using a hybrid integrated model. The curriculum needs to be an all rounded learning blueprint, where learner can improve their understanding in a progressive manner and user-friendly approach.

\subsection{AI Rules}

The AI rules define the way the online learning system assigned learning materials and exercises for the learner to follow. These are the basic rules which we have carry out in our experiments, in which we find it effective in improving the learners understanding. Online learning assessor and learner can modify all the assessment indicators accordingly (depending on various conditions and overall standard requirements) [12].

\begin{tabular}{|c|c|c|c|c|l|}
\hline $\begin{array}{c}\text { Rule } \\
\text { number }\end{array}$ & $\begin{array}{c}\text { Difficulty } \\
\text { level }\end{array}$ & $\begin{array}{c}\text { Correct } \\
\text { answers }(\%)\end{array}$ & $\begin{array}{c}\text { Understanding } \\
\text { level (\%) }\end{array}$ & $\begin{array}{c}\text { Confident } \\
\text { level (\%) }\end{array}$ & \multicolumn{1}{|c|}{ Recommendation (Response) } \\
\hline 1 & 1 & $<50$ & Nil & Nil & $\begin{array}{l}\text { Repeat the same difficulty level }=1 \\
\text { exercise }\end{array}$ \\
\hline 2 & 1 & $\geq 50$ & $<50$ & $<50$ & $\begin{array}{l}\text { Repeat the same difficulty level }=1 \\
\text { exercise }\end{array}$ \\
\hline 3 & 1 & $\geq 50$ & $<50$ & $\geq 50$ & $\begin{array}{l}\text { Repeat the same difficulty level }=1 \\
\text { exercise }\end{array}$ \\
\hline 4 & 1 & $\geq 50$ & $\geq 50$ & $<50$ & $\begin{array}{l}\text { Repeat the same difficulty level }=1 \\
\text { exercise }\end{array}$ \\
\hline 5 & 1 & $\geq 50$ & $\geq 50$ & $\geq 50$ & $\begin{array}{l}\text { Move to next difficulty level }=2 \\
\text { exercise }\end{array}$ \\
\hline 6 & 2 & $<50$ & Nil & Nil & $\begin{array}{l}\text { Repeat the same difficulty level }=2 \\
\text { exercise }\end{array}$ \\
\hline 7 & 2 & $\geq 50$ & $<50$ & $<50$ & $\begin{array}{l}\text { Repeat the same difficulty level }=2 \\
\text { exercise }\end{array}$ \\
\hline 8 & 2 & $\geq 50$ & $<50$ & $\geq 50$ & $\begin{array}{l}\text { Repeat the same difficulty level }=2 \\
\text { exercise }\end{array}$ \\
\hline 9 & 2 & $\geq 50$ & $\geq 50$ & $<50$ & $\begin{array}{l}\text { Repeat the same difficulty level }=2 \\
\text { exercise }\end{array}$ \\
\hline 10 & 2 & $\geq 50$ & $\geq 50$ & $\geq 50$ & $\begin{array}{l}\text { Move to next difficulty level }=3 \\
\text { exercise }\end{array}$ \\
\hline 11 & 3 & $<75$ & Nil & Nil & $\begin{array}{l}\text { Repeat the same difficulty level }=3 \\
\text { exercise }\end{array}$ \\
\hline 12 & 3 & $\geq 75$ & $<50$ & $<50$ & $\begin{array}{l}\text { Repeat the same difficulty level }=3 \\
\text { exercise }\end{array}$ \\
\hline
\end{tabular}




\begin{tabular}{|c|c|c|c|c|l|}
\hline 13 & 3 & $\geq 75$ & $<50$ & $\geq 50$ & $\begin{array}{l}\text { Repeat the same difficulty level =3 } \\
\text { exercise }\end{array}$ \\
\hline 14 & 3 & $\geq 75$ & $\geq 50$ & $<50$ & $\begin{array}{l}\text { Repeat the same difficulty level =3 } \\
\text { exercise }\end{array}$ \\
\hline 15 & 3 & $\geq 75$ & $\geq 50$ & $\geq 50$ & Move to next topic exercise \\
\hline
\end{tabular}

Figure 1. The AI rules applied in the experiments.

\section{Progressive Response Learning Model}

In a traditional learning model involves a teacher giving lectures to a group of students in a physical classroom. The teacher will then teach what has been prepared and planned in the curriculum. However, some students have different learning pace and understanding level. This will creates many problems not only to the teacher but to the entire class. Therefore, this Progressive Response Learning Model can min minimise these problems, where students having different learning pace and understanding level.

The principle behind this Progressive Response Learning model is 'to make those fast pace learner go fast while slow pace learner go far'. In this paper, we will present two analysis where this model can demonstrate the effectiveness when apply to online learning. In these two analysis, I will use multiple choice questions approach and the outcomes from the assessment $t$ will than be evaluated. Afterthat, the assessor and learner can easily review the online learning performance [13).

The analysis consists of 2 assessment groups from 20 voluntary online learners between the age of 9 and 10. We assigned them into 2 groups based on their school assessment results in Algebra topic, Mathematics subject, GROUP (A) - 10 fast pace learners and GROUP (B) - 10 slow pace learners. Each group will be assigned 10 Q\&A sections and each Q\&A section will have 10 questions. In total, we will apply 100 questions with the Difficulty level distribution of 400/o Low, 30\% Medium and 30\% High. I n this analysis, we will compare the traditional learning approach and the Progressive Response Leaning approach. The Q\&A questions are from Year 5: UK National Curriculum, Algebra topic (Mathematics).

\subsection{Traditional Learning Approach}

All leaners (fast and slow pace leaners) are require to do all I 0 Q\&A sections, each section a day, within 10 days. The outcomes from this assessment are represented in marks (in \%) and groups, GROUP (A) - Fast-Pace Learners and GROU P (B) - Slow Pace Learners. 

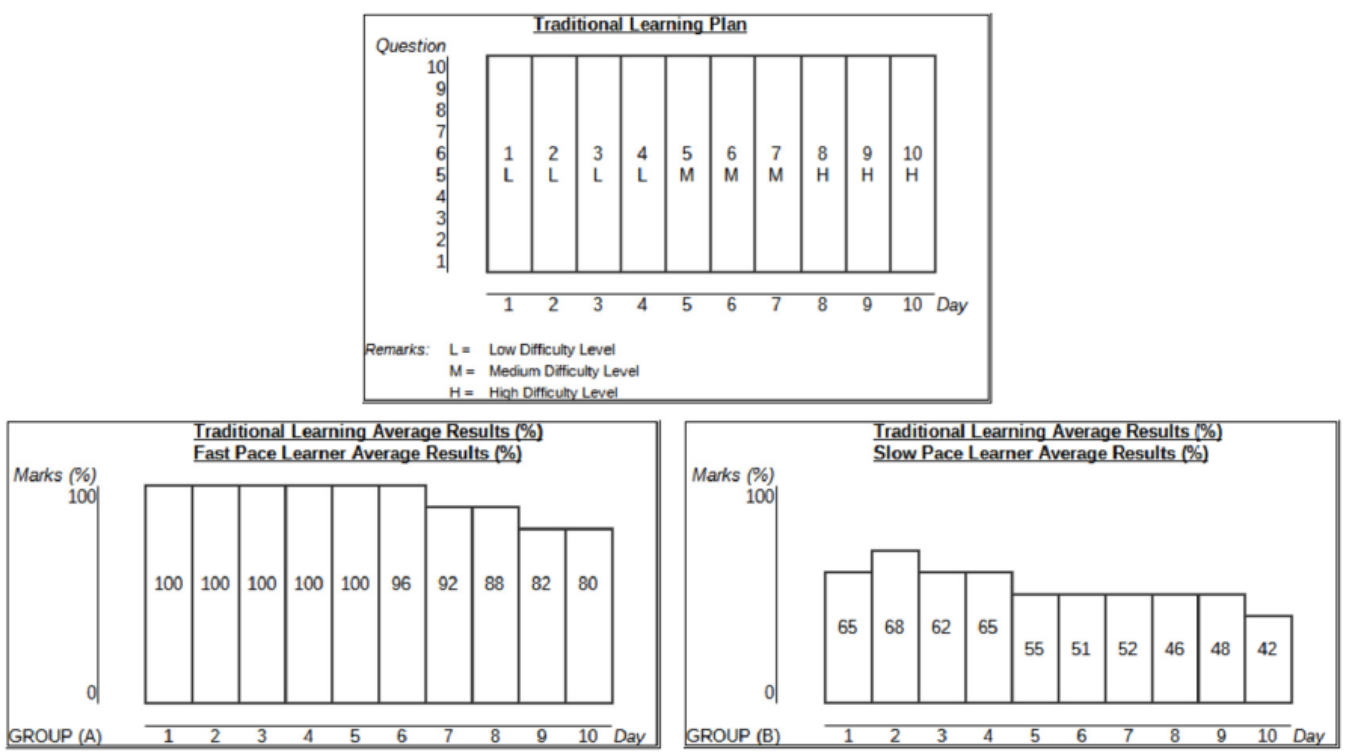

Figure 2. The results from applying traditional learning approach on two different groups of learners,

GROUP (A) - Fast Pace Learners group and GROUP (B) -Slow Pace Learners group.

\subsection{ProgressiveRespon seLearningApproach}

All leaners (fast and slow pace learners) are require to do all I 0 Q\&A sections, each section a day, within 10 days. In this model, we will repeat some of the previous Q\&A in every exercise and using this approach, we can encourage the learner to retrieve the skills the learner learned from the previous exercise and increase the confidence level when facing new quest ions. The outcomes from this assessment are represented in marks (in \%) and the Difficulty level are presented on the charts (Figure 3).
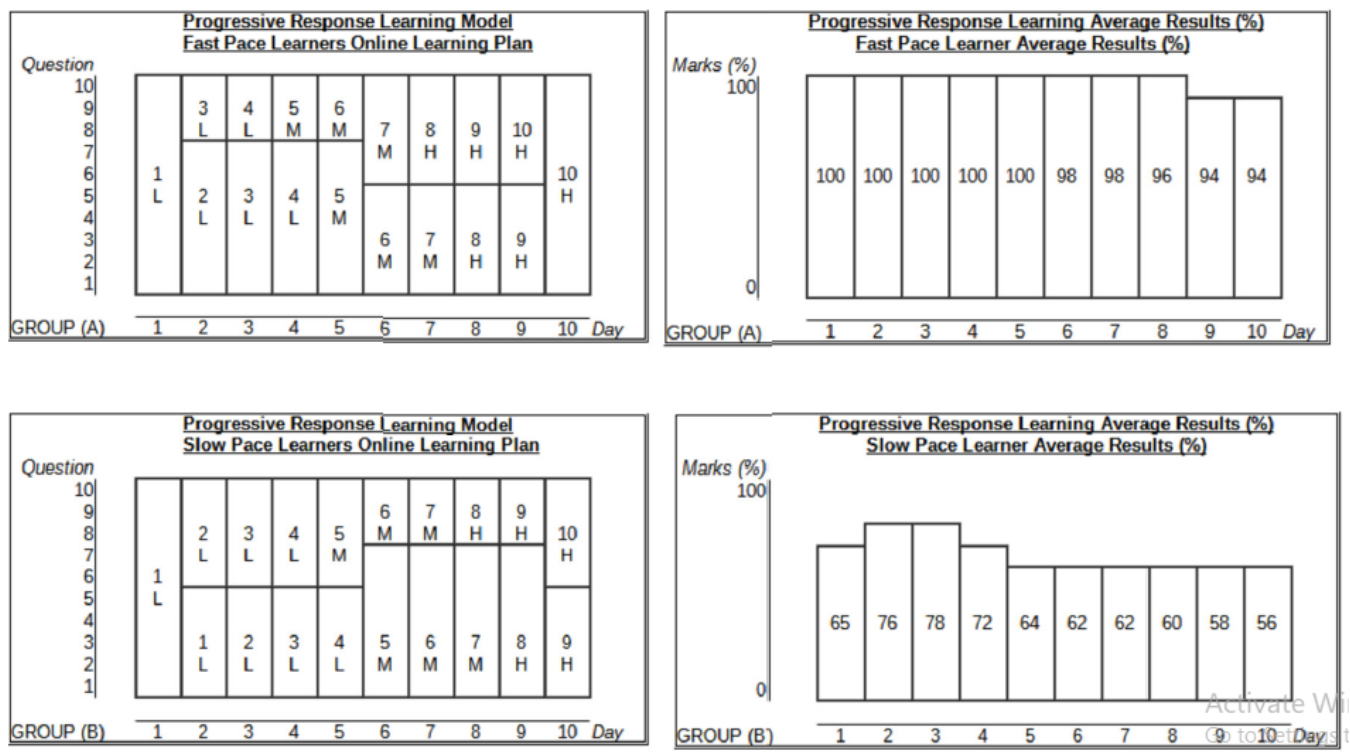

Figure 3. The results from applying Progressive Response Learning approach on twodifferent groups of learners.

GROUP (A)-Fast Pace<Leaners group and GROUP (B) -Slow Pace Learners group. 
As we can see from the preliminaryexperimental results, there are improvements in the overall marks and performance after applying the Progressive Response Learning model. Besides that, this model involves psychological factor when facing different learner's behaviour, therefore, further research and investigations are needed in this area.

\section{Online Learning System}

The online learning using $\mathrm{Al}$ system include several components, whichcan be integrated as one complete artificial intelligence online learning system (14). These are the standard components:-

1. Reasoning - It is the set of processes that empowers us to provide basis for judgement, making decisions, and prediction.

2. Learning - It is the activity of gaining in formation or skill by studying, practising, being educated, or experiencing something. Leaning improves the awareness of the subjects of the study.

3. Problem Solving - It is the procedure in which one perceives and tries to arrive at a desired solution from a current situation by taking some path, which is obstructed by known or unknown hurdles.

4. Perception - It is 1he way of acquiring, interpreting, selecting, and organizing sensory information.

5. Linguistic Intelligence - It is one's ability to use, comprehend, talk, and compose the verbal and written language. It is significant in interpersonal communication.

The potential of online learning system include 4 factors of accessibility, flexibility, interactivity, and collaboration of online learning afforded by the technology. In terms of the challenges to online learning, 6 are identified: defining online learning; proposing a new legacy of epistemology-social constructivism for all; quality assurance and standards; commitment versus innovation; copyright and intellectual property; and personal learning in social constructivism.

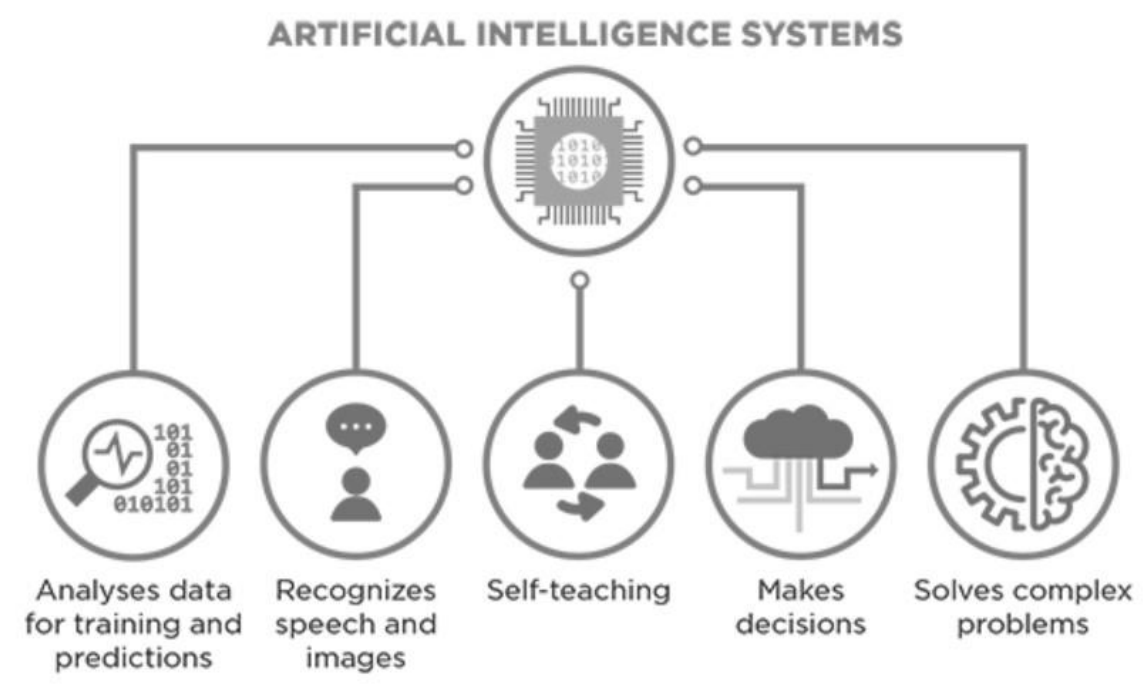

Figure 4. The artificial intelligence online learning system components.

\section{Conclusions}

Adaptive online learning is an enhancement that makes learning systems more effective by adapting the presentation of information and overall link structure to the individual learner, based 
on learners' knowledge and behaviour. The teachers can use the learner behaviour information for various analyses and make the changes in the teaching process to improve the teaching and learning process. By further expert analysis and experimentation it can become a firm educational method which uses computers as interactive teaching devices to enhance individual skills. It is based on project methodology in which learner's cognitive and psychological will be judged based on learner overall performance. It is based on AI domain and further scope of improvement is huge. The two new proposed models show promising response in AI online learning and further evaluation and research is in progress.

\section{REFERENCES}

[1] Jonathan Michael Spector, Du Jing, (2017). Artificial Intelligence and the Future of Education: Big Promises - Bigger Challenges, ACADEMICS, No. 7.

[2] Oscar Sanjuan, B. Cristina Pelayo Garcia-Bustelo, Ruben Gonzalez Crespo, Enrique Daniel France, (2009). Using Recommendation System for E-Learning Environment at degree level, International Journal of Interactive Multimedia and Artificial Intelligence, Vol. 1, No. 2.

[3] S. M. Patil, T. D. Shaikh, (2014). Implementing Adaptability in E-Learning Management System Using Moodle for Campus Environment, International Journal of Emerging Technology and Advanced Engineering, Vol. 4, No. 8.

[4] Shraddha Kande, Pooja Goswami, GurpreetNaul, Mrs. Nirmala Shinde, (2016). Adaptive and Advanced E-learning Using Artificial Intelligence, Journal of Engineering Trends and Applications, Vol. 3, No. 2.

[5] Ofra Walter, VeredShenaar-Golan and Zeevik Greenberg, (2015). Effect of Short-Term Intervention Program on Academic Self-Efficacy in Higher Education, Psychology, Vol. 6, No. 10.

[6] Calum Chace, (2019). Artificial Intelligence and the Two Singularities, Chapman \& Hall/CRC.

[7] PieroMella, (2017). Intelligence and Stupidity - The Educational Power of Cipolla's Test and of the "Social Wheel", Creative Education, Vol. 8, No. 15.

[8] Zhongzhi Shi, (2019). Cognitive Machine Learning, International Journal of Intelligence Science, Vol. 9, No. 4.

[9] Crescenzio Gallo and Vito Capozzi, (2019). Feature Selection with Non Linear PCA: A Neural Network Approach, Journal of Applied Mathematics and Physics, Vol. 7, No. 10.

[10] Charles Kivunja, (2015). Creative Engagement of Digital Learners with Gardner's BodilyKinesthetic Intelligence to Enhance Their Critical Thinking, Creative Education, Vol. 6, No. 6.

[11] EvangeliaFoutsitzi, Georgia Papantoniou, EvangeliaKaragiannopoulou, HarilaosZaragas and Despina Moraitou, (2019). The Factor Structure of the Tacit Knowledge Inventory for High School Teachers in a Greek Context.

[12] Nick Bostrom and Eliezer Yudkowsky, (2011). The Ethics of Artificial Intelligence, Cambridge University Press.

[13] Gus Bekdash, (2019). Using Human History, Psychology, and Biology to Make AI Safe for Humans, Chapman \& Hall/CRC.

[14] The Student Circles.com, Artificial Intelligence Study Notes https://www.thestudentcircle.com/quickguide.php?url=artificial-intelligence 


\section{AUTHOR}

Prof. Yew Kee Wong (Eric) is a Professor of Artificial Intelligence (AI) \& Advanced Learning Technology at the HuangHuai University in Henan, China. He obtained his BSc (Hons) undergraduate degree in Computing Systems and a Ph.D. in AI from The Nottingham Trent University in Nottingham, U.K. He was the Senior Programme Director at The University of Hong Kong (HKU) from 2001 to 2016. Prior to joining the education sector, he has worked in international technology companies, HewlettPackard (HP) and Unisys as an AI consultant. His research interests include AI, online learning, big data analytics, machine learning, Internet of Things (IOT) and blockchain technology.

(C) 2021 By AIRCC Publishing Corporation. This article is published under the Creative Commons Attribution (CC BY) license. 